\section{Effect on the Rabbit}

SMC has been considered pharmacologically inactive by Bovet et al: (1951), but nevertheless they noted a "headdrop effect" with $10 \mathrm{mg}$. of SMC iodide per $\mathrm{kg}$. of rabbit, compared with $0.2 \mathrm{mg}$. of SDC di-iodide per kg. As there was here a substance which had a pharmacological action and which could be destroyed by cholinesterases, we decided to investigate the effect on the rabbit in the first instance. The animals were females weighing between 2.5 and $3 \mathrm{~kg}$. The dosages represent active cation of SMC injected intravenously in mg. $/ \mathrm{kg}$. No effect could be seen below $0.8 \mathrm{mg}$.; 0.8 to 1.2 resulted in slight depolarizing twitches but no paralysis : 1.3 to 1.5 produced a transient head-drop; from 1.5 to 4.5 there was paralysis of increasing duration-150 seconds at 2.5 and 570 seconds at 3.5. At this latter range the breathing was shallow, but there was no apnoea. A dosage above 4.5 could, and above 5 did, regularly cause respiratory paralysis. When given in doses above $7 \mathrm{mg}$.that is, twice that producing 10 minutes' relaxation without apnoea-SMC caused a fall in blood pressure followed by a fall in heart rate. There seemed to be a hexamethoniumlike effect on the peripheral blood vessels accompanied by "pooling" of blood in dependent parts of the body. It was followed by a depressant effect on the heart, which persisted after severing the vagus. Thus there is a wide range from the head-drop dose of 1.5 to the toxic dose of 7.5. SMC is much longer acting than SDC at similar pharmacological levels-doubtless because it is so much more slowly destroyed by pseudo-cholinesterase than SDC. An injection of $1 \mathrm{ml}$. of human pseudo-cholinesterase $(16,000$ units) terminated the effect of SMC as effectively as that of SDC. In fact, such treatment will allow a rabbit (female $2.5-3 \mathrm{~kg}$.) to survive with impunity a threefold lethal dose of both SDC and SMC.

To find whether these results are applicable to man further work will have to be carried out on other mammals. One certainly would not like to embark on the injection of unusually large amounts of a choline derivative into man without ample reassurance. There exist for man a number of long-time relaxants which are readily controlled by neostigmine; nevertheless a long-acting relaxant which could be counteracted by a harmless compound might be a useful addition to the equipment of the anaesthetist. Neostigmine tends to be given in emergencies and to patients who are already at hazard, and there is always a risk of a toxic effect. Be this as it may, at any rate so far as the rabbit is concerned there exists a cholinergic compound which resembles decamethonium in its prolonged curarizing action and succinylcholine in permitting this action to be terminated at will by the injection of pseudocholinesterase.

\section{The Function of Pseudo-cholinesterase}

It has hitherto not been possible to allot to the pseudocholinesterase a function in life, particularly as, unlike true cholinesterase, it can be removed by specific inhibitors in man and animals in vivo without notable ill effect. The most important study on the distribution of pseudocholinesterase was that carried out by Ord and Thompson (1952) on brain and nerves of man and animals; they demonstrated by the interplay of specific substrates and inhibitors that the pseudo-enzyme is largely in the white matter of the central and peripheral nervous system in close association with the true cholinesterase of the grey substance. There are now at least four compounds which "poison" the true cholinesterase and are destroyed by the pseudo-cholinesterase : acetylcholine and SMC in high concentrations, SDC and suxethonium at high and low concentrations. Except for acetylcholine, the compounds tested have been synthesized in vitro, but it is obvious that similar substances must arise in the intermediary metabolism of the body. It may therefore not be unreasonable to suggest that a function of pseudo-cholinesterase is that of protecting true cholinesterase from non-specific inhibitors.

\section{Summary}

Succinylmonocholine inhibits true cholinesterase of man and is broken down by the pseudo-cholinesterase. In rabbits at concentrations of $3.5 \mathrm{mg}$. of active cation per $\mathrm{kg}$. of body weight it produces muscular relaxation of 10 minutes' duration without apnoea; its effect can be terminated at will by the injection of pseudo-cholinesterase. Prior injection of pseudo-cholinesterase will protect rabbits against toxic doses.

It is suggested that a function of pseudo-cholinesterase is that of protecting true cholinesterase against nonspecific inhibitors.

We thank Professor D. Bovet and Messrs. May and Baker Ltd. for gifts of succinylmonocholine. We are indebted to Professor K. J. Frankilin, who kindly gave us the hospitality of his laboratory for the kymographic recording of blood pressure changes; to Dr. G. Boissard, for assistance in this part of the work; and to Drs. F. T. Evans and P. W. S. Gray for helpful discussions. Dr. E. B. McLean kindly provided us with a concentrated pseudocholinesterase solution produced by a further fractionation of Cohn's fraction IV-6-1 with ammonium sulphate. We are grateful to the Medical Research Council for an expenses grant, and for a maintenance grant to one of us (E.S.).

\section{REFERENCES}

Bovet, D., Bovet-Nitti, F., Guarino, S., Longo, V. G., and Fusco, R. (1951). Arch. int. Pharmacodyn., 88,

Evans, F. 1., Gray, P. W. S., Lehmann, H., and Silk, E. (1952). Lancet. 1, 1229.

McArdle, B. (1940). Quart. J. Med., n.s. 9, 107

Ord, M. G., and Thompson, R. H. S. (1952). Biochem. J., 51, 245.

Whittaker, V. P., and Wijesundera, S. (1951). Ibid., 49, xlv.

- - (1952). Ibid. 52, 475.

\section{Medical Memorandum}

\section{Stevens-Johnson Syndrome}

A further case of this rare disease is reported in the hope that the photographs will be of help to others who have not encountered it. Involvement of the nails is a feature that has not been mentioned in previous cases.

\section{CASE Report}

A boy aged 5 years 3 months had been in good health since the removal of his tonsils and adenoids two monitis previously on account of frequent attacks of tonsillitis. The only other illnesses were measles at 3, whooping-cough at 5 , and several large epistaxes. The family history was not relevant. On September 7, 1949, a slight discharge from both eyes was noticed, and his appetite became poor. This discharge increased rapidly and the lids became oedematous. The next day his lips began to swell, a rash appeared, and he was febrile. He was admitted on Septem. ber 9 . The clinical picture is shown in the photographs, taken the next day. The features of the case were :

Bilateral angular conjunctivitis, with a profuse purulent discharge and much irritation, which made him rub the oedematous lids. There was no corneal ulceration.

Haemorrhagic crusting of the lips, strikingly confined to the red margins, extending neither to the mucous membrane inside the lips nor to the relative normal surrounding skin, producing by contrast the appearance of circumoral pallor (Fig. 1).

The Rash.-The distribution was general but more pronounced on the face, extensor surface of the forearms, palms (but not the soles), and nail margins. On the face the cheeks were the most affected, with fewer lesions round the mouth, on the forehead, and (not shown) behind the pinnae. The penis was involved no more than the trunk, and there was no urethritis. The configuration was pleomorphic, the discrete lesions varying from 2 to $5 \mathrm{~cm}$. in diameter and tending to be circular or oval. The lesions 
itched and some were in relation to scratch marks (see left forearm in Fig. 2). The early lesions were erythematopapular, later becoming vesiculo-bullous. Breaking of the bullae produced raw areas and haemorrhagic crusting. On the face the lesions were confluent. On the extensor surfaces of the forearms and on the palms, where the lesions

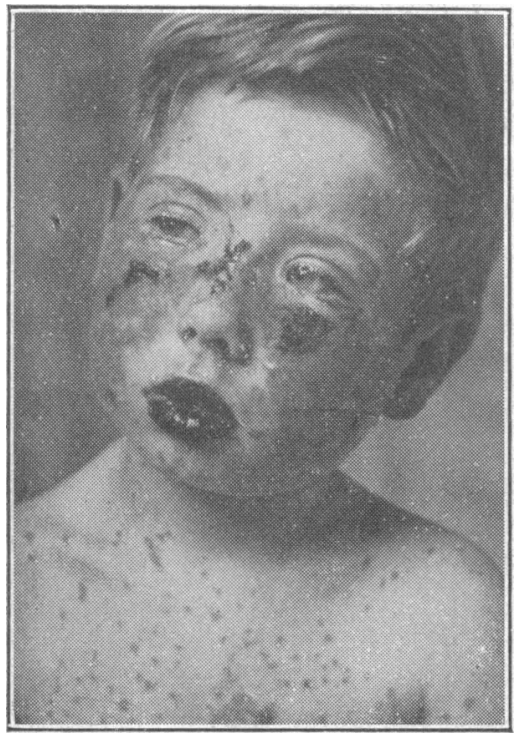

Fig. 1.

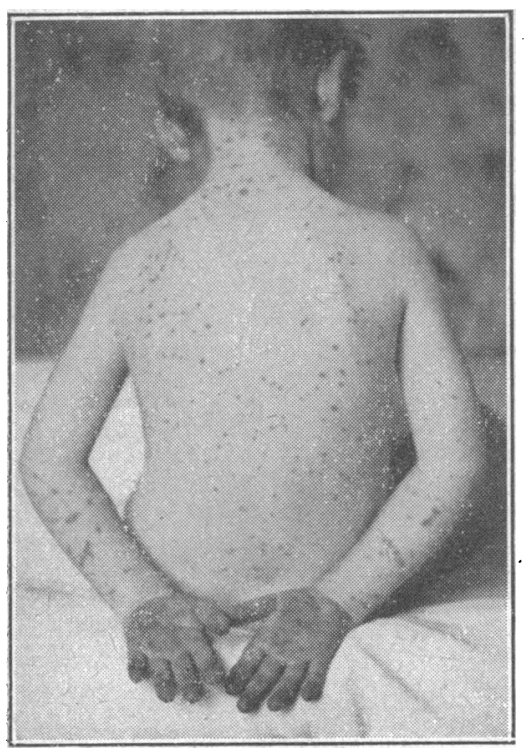

FIG. 2. we re becoming confluent, the intervening skin was e $\mathrm{r}$ y $\mathrm{t} \mathrm{h}$ e $\mathrm{m}$ atous. Elsewhere the intervening skin was normal. Oedema was confined to the cheeks, mouth, and eyelids. Except at the umbilicus, no lesion was pustular.

In the mouth there were raw areas from lesions on the mucous membranes of the cheeks; the soft palate, fauces, and tonsillar beds escaped. Surprisingly there was no enlargement of the cervical $1 \mathrm{y} \mathrm{m} \mathrm{ph}$ nodes.

The nail beds and skin of the terminal phalanges of both fingers and toes were heavily involved (Fig. 3).

On examination his temperature w a s $102.6^{\circ} \mathrm{F}$. $\left(39.2^{\circ} \mathrm{C}.\right)$ pulse 128 , and respirations 26. He was miserable and crying, but intelligent and co-operative. The lymph nodes were not enlarged. The cardiovascular and central nervous systems were normal. The respiratory system showed no evidence of infection. The liver and spleen were not palpable.

Swabs from the conjunctival, skin, and mouth lesions were taken before any treatment was given. Cultures in broth and on blood-agar and chocolate-agar $\left(+\mathrm{CO}_{2}\right)$ plates were sterile. The blood count and urine were normal.

Treatment.-(1) Aqueous penicillin, 100,000 units sixhourly for six days, then procaine penicillin, 300,000 units (1 ml.) daily for a further six days. (2) Tab. sulphadimidin. 1 g. six-hourly for six days (total 24 g.). (3) Penicillin cream to the broken bullae. (4) The eyes were irrigated every six hours with $1 \%$ boric eye lotion, after which penicillin eye drops $(1,000$ units per ml.) were instilled. (5) Tab. phenobarbiton., $\frac{1}{2}$ gr. ( $32 \mathrm{mg}$.) twice daily, during the first ten days.

The patient was apyrexial and the pulse rate became normal within 24 hours. There was no further rise in temperature. Also, by the next day, in spite of the alarming appearance, he was feeling well and faced the camera with co-operation and composure. In a week the conjunc- tivitis had cleared up completely. After a fortnight all the skin lesions had cleared except for a little dry crusting where the larger bullae had been. He was discharged home in good health on October 10 after 31 days in hospital. When seen in January, 1950, he had remained in good health. He had shed all the nails of his fingers and toes twice since discharge from hospital. In November, 1950, he was well and there has been no recurrence of any skin lesions.

\section{COMMENT}

This disease was reviewed by Thomas (1950), who regarded it as a severe form of erythema exudativum multiforme (Hebra). Thomas stated : "It occurs to me that simpler appellations would be 'erythema multiforme exudativum major' and 'erythema multiforme exudativum minor." Although possibly attractive to the purist, most doctors will prefer to leave this esoteric sesquipedalianism to their dermatological colleagues and continue to call the disease the Stevens-Johnson syndrome.

The clinical picture is pathognomonic and must rank with diseases such as cretinism, mongolism, pink disease, etc., which, once seen, can thereafter be recognized at a glance.

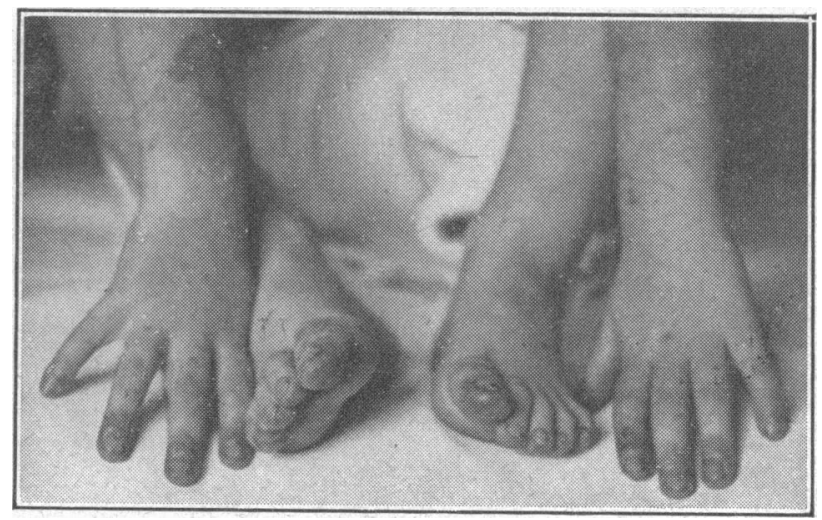

Fig. 3.

This case satisfied the criteria of the Stevens-Johnson syndrome-namely, a generalized eruption, continued fever (cut short by treatment), inflamed buccal mucosa, and severe purulent conjunctivitis. The marked involvement of the nail beds, with subsequent shedding of the nails twice, is not mentioned in other cases. This boy was never very ill, and within 24 hours of admission became apyrexial, improving steadily thereafter. The dangerous complications of corneal ulceration and pneumonia never looked likely to occur, and gave no anxiety. This prompt improvement must be attributed to the energetic treatment, which prevented any secondary infection. It seems to me that, in these days when sulphonamides are more of a food than a medicine, it is difficult to be sure that toxicity to sulphonamides can be a cause of the Stevens-Johnson syndrome. Certainly, except in persons known to be sulphonamidesensitive, such a consideration should not prevent sulphonamides being given in full doses to prevent secondary infection.

For permission to publish this case I am grateful to Dr. Duncan Brierley, the family doctor, and to Dr. W. H. Macmillan, under whose care the boy was while in hospital. I am indebted to $\mathrm{Mr}$. J. Parkes Foy for the photographs.

C. ROMER, M.B., B.S.

REFERENCE

Thomas, B. A. (1950). British Medical Journal, 1, 1393.

The first party of British children to go to Denmark this year under the Anglo-Danish Sanatorium Scheme for British Children (see Journal, 1952, 1, 634) left Northolt Airport recently. The party included a number of Scottish children and also some from Liverpool. About 20 children who have been at Vordingborg Sanatorium through this scheme for several months returned to England on the same day. 